\title{
Etude de la minéralomasse et du cycle biologique dans deux peuplements de Pin laricio de Corse, dont l'un a été fertilisé à la plantation
}

\section{J. RANGER}

Avec la collaboration de Dominique Ranger, Louisette Masar, Dominique Vatrelles

I.N.R.A.,

Station de Recherches sur les Sols forestiers et la Fertilisation

Centre de Recherches de Nancy

Champenoux, 54280 Seichamps

\section{Résumé}

Les résultats d'une étude de l'influence de la fertilisation sur les modifications des biomasse, minéralomasse et cycle biologique en jeune plantation résineuse dans l'ouest de la France (forêt domaniale de Moulière, Vienne), sont présentés dans cet article. Deux écosystèmes sont comparés, un peuplement de Pin laricio de Corse (Pinus nigra Arn., ssp laricio Poiret) ayant reçu une fertilisation complète à la plantation (1959) et un peuplement témoin non fertilisé

A côté des évaluations statiques - biomasse, minéralomasse, réserves du sol - on a cherché à étudier les flux entre les différents compartiments des écosystèmes; on a ainsi évalué : l'immobilisation courante annuelle de tels peuplements, les restitutions au sol par les chutes de litières et les pluviolessivats, les prélèvements annuels nets de bioéléments par les peuplements en apportant quelques précisions sur l'évaluation des transferts internes d'éléments nutritifs.

\section{1. - But de l'étude}

«La capacité de production des écosystèmes forestiers est surtout liée à la masse d'éléments nutritifs présents dans le sol. Le contrôle de la production implique l'obtention de renseignements sur la quantité d'éléments contenue dans le sol et sur le cycle biogéochimique, ainsi que les modifications liées aux différentes pratiques d'utilisation et d'exploitation des forêts. » Cet extrait du rapport 1974 du M.A.B., au sujet des forêts feuillues et des forêts mixtes tempérées situe bien le cadre de notre étude. Celle-ci avait pour but de préciser l'influence d'une fertilisation sur la modification des biomasses, des minéralomasses et du cycle biologique en forêt. Nous avons comparé deux écosystèmes constitués l'un par un peuplement de Pin laricio de Corse (Pinus nigra Arn ssp laricio Poiret) ayant reçu une fertilisation complète à la plantation et l'autrc par un peuplement témoin non fertilisé. 


\section{2. - Généralités sur les stations étudiées}

L'étude a été réalisée en forêt domaniale de Moulière (Vienne) située à une vingtaine de kilomètres au Nord de Poitiers dans une expérience de fertilisation installée en 1959 et décrite par M. Bonneau (1963, 1970).

Nous avons travaillé sur deux traitements, une fertilisation complète et un témoin dans un seul bloc ( $\left.\mathrm{n}^{\circ} \mathrm{III}\right)$. La présentation écologique complète des peuplements a été faite dans un article consacré à l'étude des biomasses (RANGER, 1977).

Rappelons les caractéristiques essentielles des traitements étudiés :

Traitement fertilisé $8.0(F)$ :

- la fertilisation : $92 \mathrm{~kg}$ d'azote (urée), $380 \mathrm{~kg}$ de phosphore $\left(\mathrm{P}_{2} \mathrm{O}_{5 .}\right), 800 \mathrm{~kg}$ de chaux $(\mathrm{CaO})$ et $160 \mathrm{~kg}$ de potasse $\left(\mathrm{K}_{2} \mathrm{O}\right)$ enfouis par labour à l'automne 1959 ;

- la plantation : Pin laricio de Corse associé à $1 / 6$ de feuillus (chêne rouge d'Amérique), densité de plantation 4500 plants/ha.

Traitement témoin $3.0(\mathrm{~T})$ :

- pas de fertilisation ;

- plantation identique à $(F)$ après labour.

Les sols de type lessivés à pseudogley sont lourds, pauvres et mal drainés. La fertilisation produit un doublement de la production primaire (en y incluant pour le peuplement fertilisé les produits de l'éclaircie sanitaire réalisée en 1973) de l'origine à 15 ans.

\section{3. - Matériel et méthodes analytiques}

\section{1. - Le matériel végétal}

\subsection{1. - L'échantillonnage}

- Les arbres forestiers : la publication sur la biomasse fait le point sur l'échantillonnage. Le matériel végétal recueilli (en octobre 1975) pour la détermination des biomasses sert à la détermination des minéralomasses. C'est le cas également pour la végétation concurrente des arbres forestiers.

- Les retombées annuelles de litière : sous chaque peuplement sont répartis au hasard sur toute la surface six pièges à litière rectangulaires de $0,1365 \mathrm{~m}^{2}$ de surface. Ces pièges sont constitués par des bacs en p.v.c. posés sur le sol. La litière est piégée par une toile inaltérable située à mi-hauteur du bac, ce qui permet d'éviter une évolution trop poussée des débris organiques entre les prélèvements.

Le nombre de bacs permet d'évaluer avec suffisamment de précision les chutes de litière issues de la couronne des arbres. Cependant les débris végétaux issus de l'exfoliation des écorces des troncs ne sont pas pris en compte.

Les prélèvements ont été effectués tous les mois la première année (automne 1976, automne 1977), puis d'une façon plus espacée la deuxième année.

\subsection{2. - Le conditionnement des échantillons}

Les échantillons sont mis à sécher à l'étuve à 65 "C puis broyés, homogénéisés et stockés en flacons étanches. 


\subsection{3. - Analyse chimique}

$\mathrm{N}$ : minéralisation Kjeldahl modifiée et dosage à l'autoanalyseur Technicon.

$\mathrm{P}, \mathrm{K}, \mathrm{Ca}, \mathrm{Mg}$ : minéralisation par voie humide en milieu oxydant $\left(\mathrm{H}_{2} \mathrm{O}_{2}+\mathrm{HClO}_{4}\right)$.

Dosage $\mathbf{P}$ : autoanalyseur Technicon (colométrie du complexe phosphovanadomolybdique) quand les teneurs sont élevées, sinon dosage céruléomolybdique (colorimétrie du complexe phosphosulfomolybdique).

$\mathrm{K}, \mathrm{Ca}, \mathrm{Mg}$ : dosage par spectrophotométrie d'absorption atomique.

\section{2. - Les sols}

\subsection{1. - Les prélèvements}

Dans chaque peuplement 10 profils de sol ont été prélevés en séparant la litière et l'horizon organique très humifère, puis on fait des prélèvements systématiques tous les $10 \mathrm{~cm}$ jusqu'à $60 \mathrm{~cm}$ de profondeur. La densité apparente est mesurée par la méthode du cylindre ( 2 répétitions pour chacun des horizons et pour chaque profil).

\subsection{2. - Le conditionnement des échantillons}

Les échantillons sont mis à sécher à l'étuve à $45^{\circ} \mathrm{C}$ puis tamisés, broyés à 200 «et stockés en flacons étanches.

\subsection{3. - Les analyses des éléments totaux}

Le carbone organique : méthode WALKLEY \& BLACK (1934).

L'azote total : méthode Kjeldahl modifiée, dosage à l'autoanalyseur technicon.

Le phosphore : dosage céruléomolybdique après fusion alcaline au carbonate de sodium.

$\mathrm{Ca}, \mathrm{Mg}, \mathrm{K}$ : dosage à l'absorption atomique.

\subsection{4. - Analyse des éléments échangeables et assimilables}

P : méthode Duchaufour-Bonneau (1959).

$\mathrm{K}$ et $\mathrm{Ca}$ : extraction à l'acétate d'ammonium à $\mathrm{pH} 7$, dosage par absorption atomique.

\section{3. - Les eaux de drainage}

Il ne nous a pas été possible de mesurer le drainage in situ dans ce type de sol, c'est pourquoi il a été évalué à partir de 3 lysimètres par peuplement, constitués par des monolithes de terre en place de $60 \mathrm{~cm}$ de haut et $30 \mathrm{~cm}$ de diamètre prélevés sur le terrain par percussion. Ces lysimètres sont munis d'un dispositif de drainage qui permet de recueillir les eaux dans des bidons placés à l'abri de la lumière. Ces lysimètres sont placés en serre et arrosés au prorata de la pluviométrie moyenne mensuelle avec de l'eau déminéralisée.

Les eaux sont recueillies mensuellement et analysées après concentration par évaporation (8 fois).

P : minéralisé par $\mathrm{H}_{2} \mathrm{O}_{2}$ et $\mathrm{HClO}_{4}$, dosage par la technique céruléomolybdique.

$\mathrm{Ca}, \mathrm{Mg}, \mathrm{K}$ : même minéralisation et dosage par absorption atomique. 


\section{4. - Les eaux de lessivage des cimes}

Deux pluviomètres sont placés sous chaque couvert forestier et sont relevés mensuellement. Le volume est mesuré et les analyses sont faites comme pour les eaux de drainage.

Un pluviomètre est placé en dehors du couvert forestier, mais vraisemblablement trop près des peuplements, de plus il a été pollué. Les relevés utilisés sont ceux du pluviomètre de la Maison Forestière distante de seulement un kilomètre de l'expérience.

\section{4. - La minéralomasse d'un peuplement forestier}

\section{1. - Généralités}

La minéralomasse représente la masse d'éléments minéraux retenus dans la biomasse (nous y incluons l'azote). Elle provient de l'accumulation par les plantes des substances minérales puisées dans le sol (Duvigneaud, 1974).

La détermination de la minéralomasse du peuplement forestier suppose la connaissance aussi précise que possible de la biomasse stratifiée en compartiments de composition chimique homogène. Un soin particulier doit être pris pour déterminer les petits compartiments (feuilles par âge, rameaux séparés en écorce et bois...), car leurs teneurs en éléments minéraux est généralement très supérieure à celle des organes de plus forte biomasse. C'est à partir de cette observation que nous avons conçu un protocole assez complexe d'échantillonnage de biomasse. Nous ne reviendrons pas sur ce protocole décrit en détail dans une publication (RANGER, 1977).

La teneur retenue pour un compartiment donné est la teneur moyenne résultant des analyses faites sur les 3 arbres échantillonnés.

Les compartiments suivants ont été analysés :

- les troncs : analyse séparée pour l'écorce et le bois sur une rondelle prise à la base de chaque tronçon d'un mètre. La teneur moyenne pour le tronc s'obtient en pondérant la teneur de chaque rondelle à ses dimensions $\left(\mathrm{d}^{2}\right.$, e) ;

- les racines : analyse séparée pour l'écorce et le bois et par classe de diamètre sur des échantillons moyens par arbre;

- les branches vivantes : analyses séparées pour les feuilles, le bois, l'écorce par pousse annuelle et par niveau de stratification dans la couronne sur des échantillons moyens issus du regroupement des 4 échantillons orientés de biomasse;

- les branches mortes : analyse séparée pour l'écorce et le bois sur des échantillons moyens regroupant les 4 directions.

La minéralomasse s'obtient en multipliant la teneur moyenne d'un compartiment par sa biomasse.

\section{2. - La minéralomasse des deux peuplements après 15 ans de végétation}

Les résultats sont consignés dans les tableaux suivants :

- peuplement fertilisé : tableau 1 ;

— peuplement témoin : tableau 2. 


\subsection{1. - Variations qualitatives des éléments minéraux majeurs}

Un certain nombre de lois générales se dégagent :

- Les différents compartiments s'ordonnent quant aux teneurs en fonction de l'élément considéré :

- pour NPK : feuilles $\gg$ écorce $>$ bois ;

- pour $\mathrm{Mg}, \mathrm{Ca}$ : feuilles \# écorce $>$ bois.

- La composition minérale des tissus évolue avec l'âge : pour les feuilles il s'agit d'un vieillissement réel; pour le bois et l'écorce, le vieillissement n'intéresse qu'un certain pourcentage de l'organe qui a été analysé globalement, ce qui tamponne le phénomène. Quand ils vieillissent :

- les feuilles s'appauvrissent en $\mathbf{N}, \mathbf{P}, \mathrm{K}, \mathrm{Mg}$ et se chargent en Ca ;

- les écorces subissent les mêmes variations jusqu'à l'âge de 4 ans environ, ensuite elles s'appauvrissent en tout élément. Tant que la quantité de tissu vivant l'emporte c'est elle qui implique les variations; au-delà de 4 ans la nécromasse l'emporte ;

- les bois s'appauvrissent en tout élément.

- La composition minérale d'un tissu varie avec la hauteur relative du compartiment dans l'arbre.

L'élément le plus facile à interpréter est le calcium : dans tous les organes (feuilles, bois, écorce) sa teneur augmente quand l'altitude relative des tissus analysés diminue. Le magnésium a un comportement semblable mais moins net. L'azote, le phosphore et le potassium auraient un comportement inverse.

La fertilisation induit des différences de teneur entre les organes comparables des deux peuplements qui peuvent se résumer de la façon suivante :

- pour $\mathbf{N}$ et $\mathrm{K}$ : pas de différence significative;

- pour $\mathrm{Ca}$ et $\mathrm{Mg}$ : les tissus des arbres fertilisés sont légèrement plus riches que ceux des arbres témoins;

- pour P les tissus des arbres fertilisés sont beaucoup plus riches que ceux des arbres témoins. Ce n'est pas surprenant car la fertilisation a amélioré nettement le statut nutritif des arbres en phosphore dans un sol particulièrement pauvre en cet élément.

\section{Cas particulier du bois de tronc}

Les troncs des arbres moyens des peuplements $\mathrm{F}$ et $\mathbf{T}$ ont été analysés systématiquement mètre par mètre et cerne par cerne. Le but est de connaître la composition minérale du bois en fonction de l'âge et de la position relative dans l'arbre, et l'effet de la fertilisation sur l'immobilisation.

La répartition spatiale des éléments est assez semblable dans les deux cas :

- Pour P, K, Mg on a distingué 4 zones :

- la zone apicale la plus riche,

- la zone externe ( 2 ou 3 cernes extérieurs) encore assez riche avec des teneurs qui décroissent régulièrement du haut vers le bas de l'arbre,

- la zone centrale pauvre dans laquelle les teneurs restent à peu près constantes,

- le cerne central toujours plus riche que les cernes correspondants à un niveau donné. 







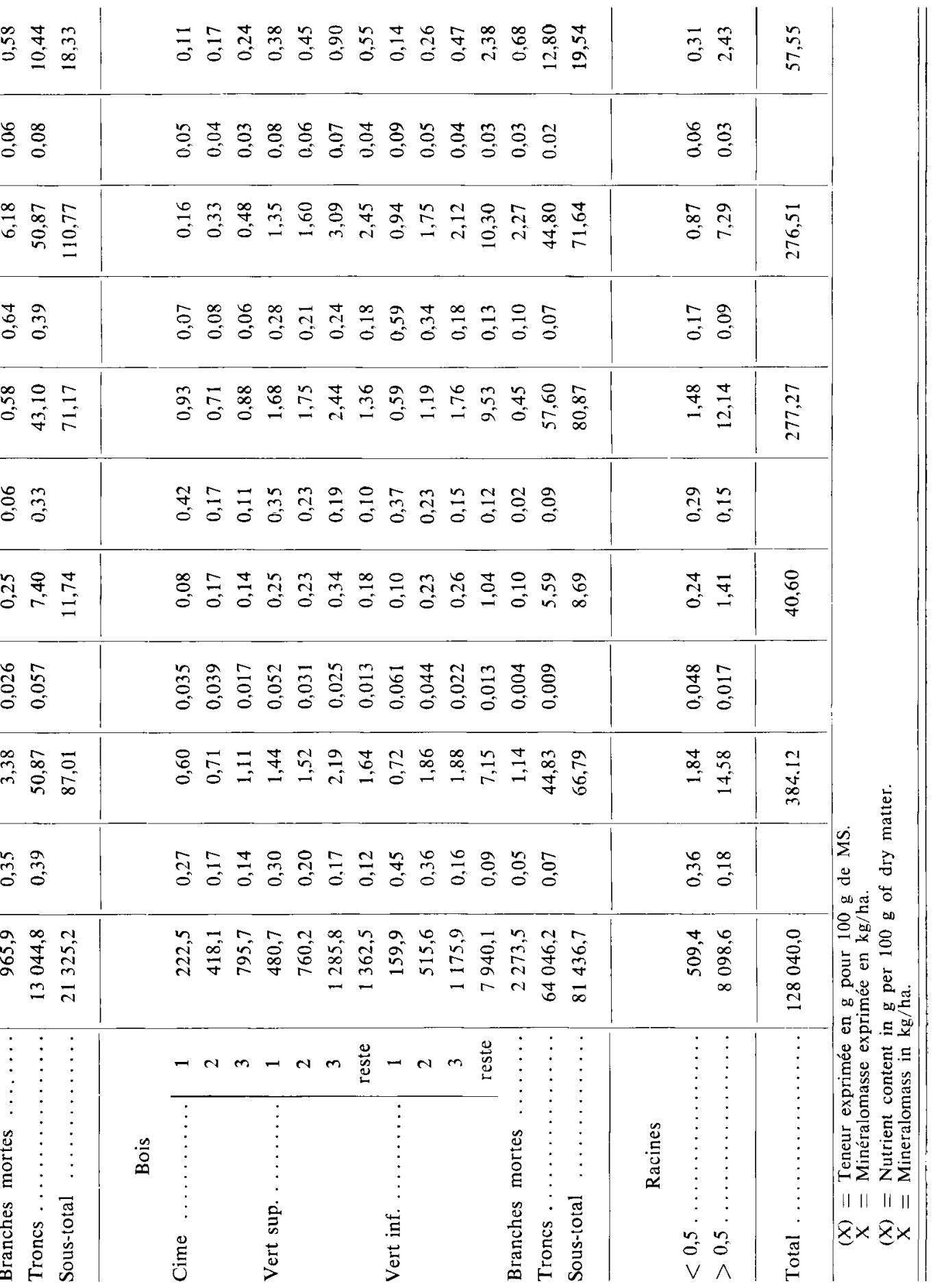




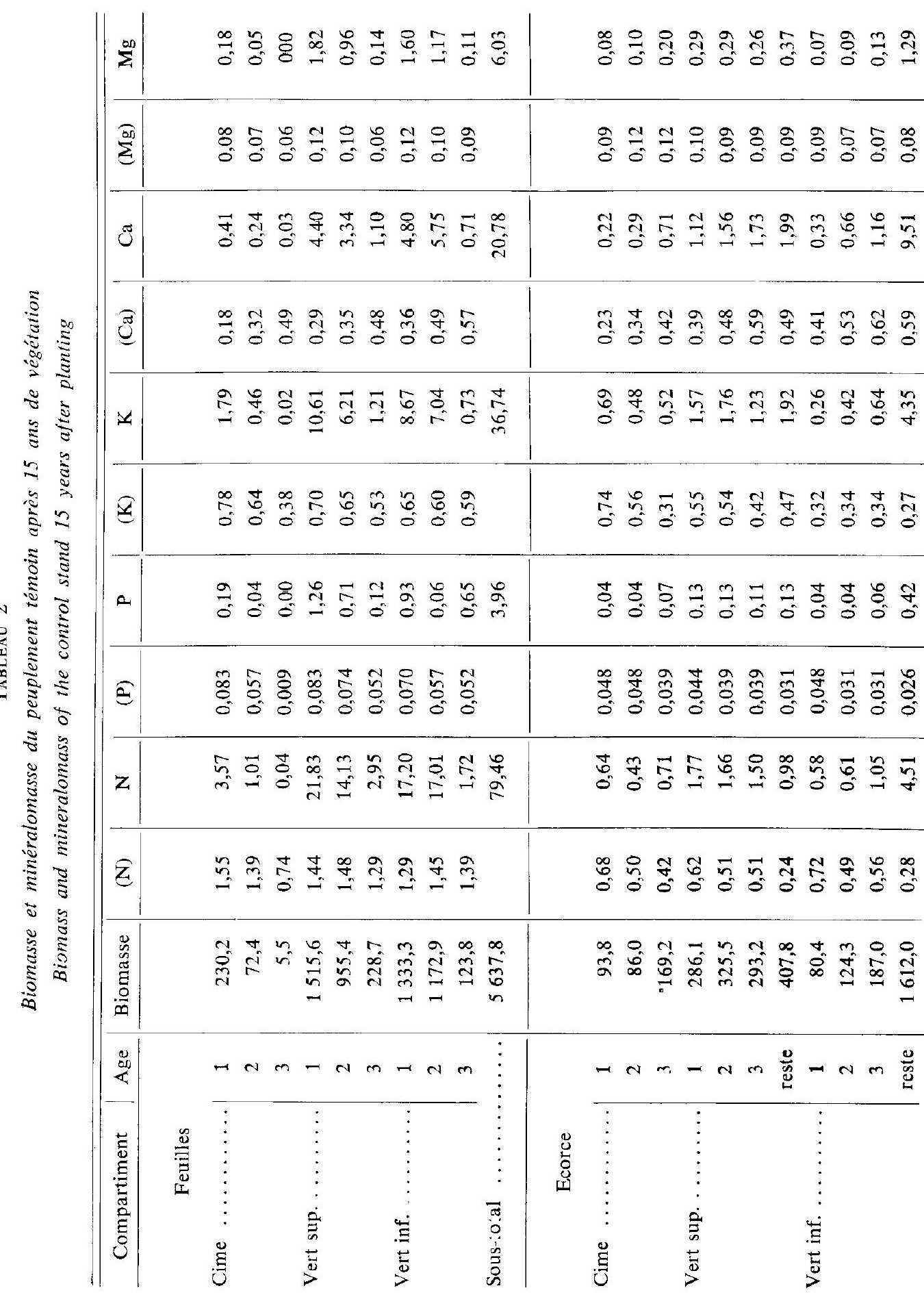




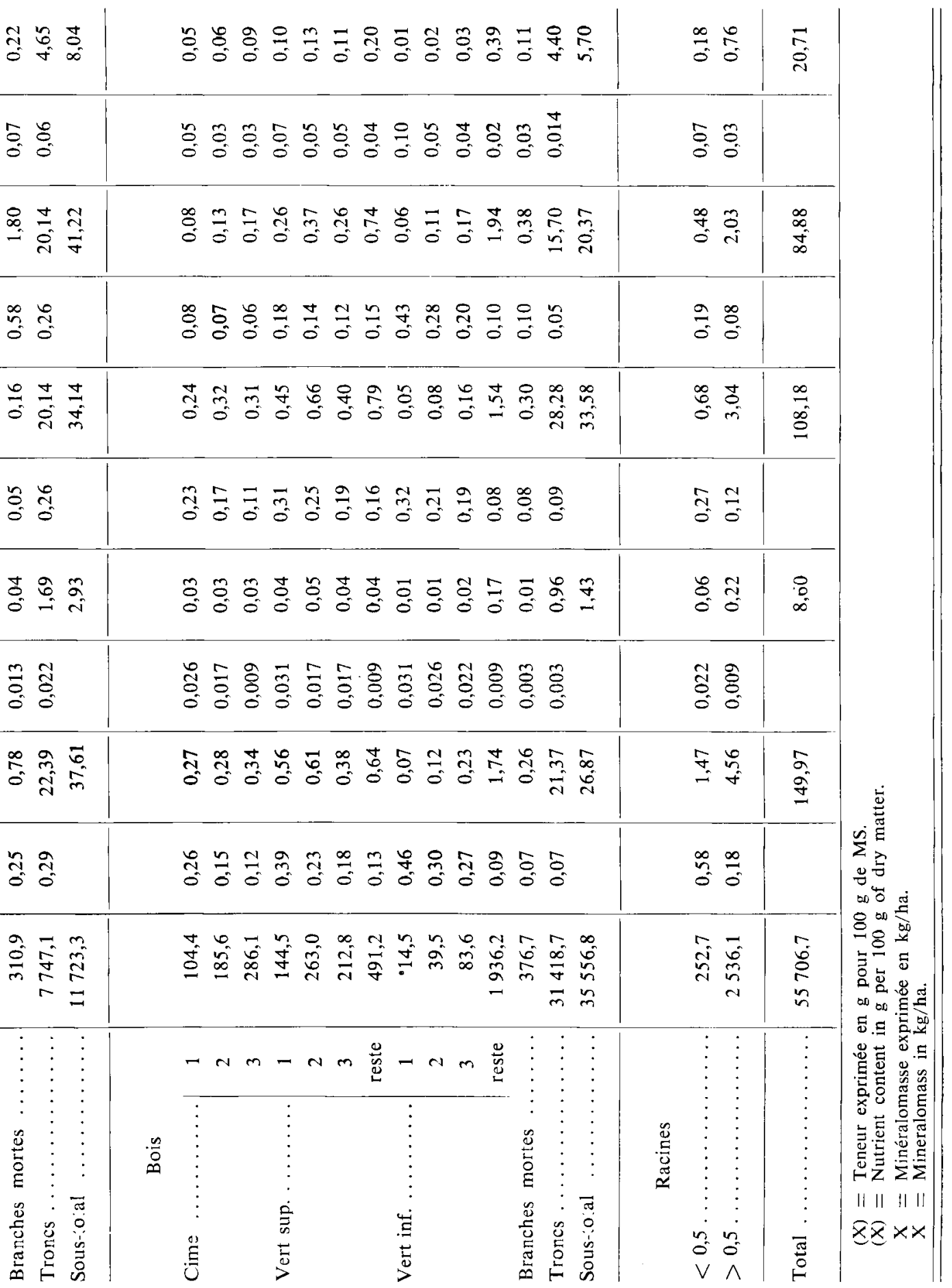


- Pour le calcium on n'observe qu'une stratification verticale plus ou moins nette. La teneur à un même niveau reste assez constante, seul le cerne central est toujours plus riche que les autres.

L'arbre fertilisé présente en outre pour le phosphore une stratification verticale des teneurs beaucoup plus nette que l'arbre témoin. C'est cet élément qui matérialise le mieux les zones fonctionnelles décrites par CLÉment et al. (1973) et les étapes de vieillissement du bois.

\subsection{2. - Variations quantitatives des éléments majeurs (tableaux 1 et 2)}

On constate que le paramètre biomasse impose le plus souvent son effet sur la quantité d'éléments retenus dans un compartiment. Cependant on voit combien il est important de ne pas négliger les petits compartiments pour l'évaluation des minéralomasses : par exemple les troncs qui représentent 60 à $70 \mathrm{p}$. cent de la biomasse totale ne retiennent que 30 à 32 p. 100 du phosphore total de la minéralomasse, alors que les aiguilles, qui ne représentent que 10 à 11 p. 100 de la biomasse retiennent 45 p. 100 du phosphore.

La différence entre les deux peuplements est importante. Le rapport $\frac{F-T}{T}$ global est toujours compris entre 150 et 400 p. 100 , ce qui montre que la fertilisation a produit un accroissement qualitatif et quantitatif des minéralomasses puisque le même rapport pour la biomasse ne dépasse pas 130 p. 100.

$\mathrm{Si}$ on fait le rapport entre la minéralomasse totale du peuplement et le volume du bois de tronc, on peut apprécier la quantité apparente d'éléments minéraux nécessaire à la fabrication d'un $\mathrm{m}^{3}$ de bois à 15 ans. Nous avons retenu la valeur de 0,4 pour la densité du bois de Pin laricio (tableau 3).

\section{Tableau 3}

Quantité apparente d'éléments minéraux exprimée en $\mathrm{kg} / \mathrm{ha}$, nécessaire à l'élaboration d'un $\mathrm{m}^{3}$ de bois de tronc à 15 ans

Apparent quantity of mineral elements (in $\mathrm{kg} / \mathrm{ha}$ ) necessary to the elaboration of one $\mathrm{cm}$ of bole wood at age 15

\begin{tabular}{c|c|c|c|c|c|c}
\hline \hline & $\begin{array}{c}\text { Volume } \\
\text { sous écorce }\end{array}$ & $\mathrm{N}$ & $\mathrm{P}$ & $\mathrm{K}$ & $\mathrm{Ca}$ & $\mathrm{Mg}$ \\
\cline { 2 - 6 } Fertilisé ....... & 160 & 2,17 & 0,23 & 1,60 & 1,63 & 0,34 \\
Témoin ...... & 79 & 1,96 & 0.11 & 1,39 & 1,08 & 0,26 \\
\hline
\end{tabular}

On s'aperçoit que l'efficience des éléments est nettement moins importante dans le peuplement fertilisé que dans le témoin, elle est même deux fois moins forte pour le phosphore. Si on effectue les mêmes calculs en ne tenant compte que du phosphore immobilisé dans les troncs $(13,0 \mathrm{~kg}$ dans $\mathbf{F}$ contre 2,6 dans $\mathrm{T}$ ), on retrouve la même différence, ce qui pose le problème d'une certaine «consommation de luxe». En 
fait l'immobilisation est faible et une grande partie des bioéléments revient au sol par le cycle biologique.

4.23. - Variations quantitatives des éléments minéraux majeurs dans la végétation totale

Dans le peuplement témoin, dont le couvert n'est pas fermé, se développe une végétation intense composée essentiellement de Callune (Calluna vulgaris), de brande (Erica scoparia), d'ajonc nain (Ulex nanus) et de fougère aigle (Pteris aquilina). La biomasse de cette végétation concurrente est de $40 \mathrm{t} /$ ha de matière sèche. Elle retient une masse importante d'éléments nutritifs qui est approximativement équivalente à la minéralomasse du peuplement forestier du témoin.

La différence de minéralomasse totale entre les deux peuplements est consignée dans le tableau 4. On constate que le bilan est excédentaire pour le placeau fertilisé en tout élément, sauf en azote.

\section{TABLEAU 4}

Différence entre les minéralomasses (exprimées en $\mathrm{kg} / \mathrm{ha}$ ) des végétations totales des deux peuplements à 15 ans

Difference between the mineralomasses (in $\mathrm{kg} / \mathrm{ha}$ ) of total vegetations of the two stands (15 years old)

\begin{tabular}{r|c|c|c|c|c}
\hline & $\mathrm{N}$ & $\mathrm{P}$ & $\mathrm{K}$ & $\mathrm{Ca}$ & $\mathrm{Mg}$ \\
\hline $\begin{array}{r}\text { Minéralomasse de la végétation } \\
\text { concurrente du témoin ... }\end{array}$ & 377,1 & 12,3 & 62,3 & 76,7 & 22,4 \\
$\begin{array}{r}\Delta(\mathrm{F}-\mathrm{T}) \text { pour l'ensemble de la } \\
\text { végétation ............... }\end{array}$ & $-143,0$ & 19,7 & 106,8 & 114,9 & 14,4 \\
\hline
\end{tabular}

\section{5. - Le cycle des éléments nutritifs}

\section{1. - Généralités}

De très nombreux auteurs se sont intéressés à l'étude du cycle des éléments nutritifs dans les écosystèmes forestiers, citons : ReNnie (1955), Duvigneaud et al. (1964), Ovington (1965 et 1971), Young (1965), Rodin \& Bazilevich (1967), Rapp (1967, 1969, 1971), Aussenac et al. (1972), Ulrich (1973), Mälkonen (1974), ArNEtT et al. (1977) la majorité de ces études concernent les écosystèmes résineux.

Les études théoriques sont bien avancées; cependant comme le signale ULRICH (1973) le manque de données de base pour les divers types forestiers limitera vraisemblablement l'évolution dans ce domaine. 
La dynamique d'un écosystème est très complexe comme le montre l'examen des flux d'énergie entre compartiments (Duvigneaud, 1974; UlRICH, 1973). Nous travaillons sur un modèle simplifié qui ne prend pas en compte la production primaire brute, mais la production primaire nette. Ces deux données sont liées par la relation : trophes

Production primaire brute $=$ production primaire nette + respiration des auto(la productivité représente la production annuelle).

Le cycle biologique représente la circulation des éléments biogènes au sein de l'écosystème. Ce cycle appartient au cycle général des éléments minéraux dans la biosphère : le cycle géochimique.

Le cycle biologique annuel se décompose comme suit (d'après ULRICH, 1973 ; Duvigneaud, 1974) :

- le prélèvement (ou absorption) d'éléments par les racines des plantes;

- le retour (ou restitution) au sol par la chute des litières, les eaux de lavage des phytocénoses, les exsudats racinaires et les racines mortes;

- l'immobilisation (ou rétention) nécessaire à l'accroissement des organes pérennes de la phytocénose (nous serons amenés à préciser cette définition).

Au niveau de l'arbre (ou du peuplement) la relation entre les différents points est la suivante :

$$
\text { prélèvement }=\text { immobilisation }+ \text { retour }
$$

Cette équation n'est toutefois valable que pour un écosystème équilibré, ce qui n'est pas le cas de notre étude.

L'équation devient alors :

(1) prélèvement $=$ immobilisation + variation de la masse d'éléments nécessaires à l'accroissement des feuilles (*) et radicelles + retours nets.

Une autre équation permet de chiffrer le prélèvement :

(2) prélèvement $=$ immobilisation + minéralomasse des feuilles de l'année - transferts internes.

Nous nous sommes intéressés au problème des transferts internes qui représentent une source importante d'éléments nutritifs pour les plantes pérennes. Celle-ci provient de la migration d'élément (surtout NPK) des parties âgées vers les parties jeunes de la plante. Il s'agit de processus complexes qui concernent les échanges d'éléments entre feuilles âgées et bois puis entre bois et feuilles jeunes d'une part et entre bois âgé et bois et feuilles jeunes d'autre part. transferts :

A partir des équations précédentes on peut donner une évaluation de ces

(3) transferts internes $=$ minéralomasse des feuilles de l'année - (variation de la masse d'éléments nécessaires à l'accroissement des feuilles et radicelles + retours nets).

$\left({ }^{*}\right)$ Il s'agit ici du compartiment «feuille » pris globalement. 
Dans la mesure où nous voulons chiffrer de manière relativement simple les grands composants du cycle, l'immobilisation indiquée dans les formules (1) et (2) doit être facilement mesurable, c'est-à-dire une immobilisation nette (transferts des feuilles vers le bois, ou fixation directe d'éléments dans les racines, diminuées du transfert du bois vers les feuilles jeunes).

Dans ces conditions les transferts indiqués dans ces formules ne représentent que les transferts des feuilles âgés vers les feuilles jeunes.

Nous arrivons à la même formulation que celle de Bringmark (1974). Mais il peut être intéressant d'aller davantage dans le détail et d'essayer de chiffrer aussi les transferts qui existent des bois et des écorces vers les organes en croissance (feuilles - rameaux) ainsi que les évoquent WeLls \& JöRgENSEN (1975) et MäLKöNEN (1975). Il faut pour cela connaître d'une part l'évolution qualitative des compositions chimiques des divers tissus en fonction du temps et d'autre part la productivité ligneuse courante des peuplements. La quantité d'éléments impliquée dans les transferts issus des bois et écorce sera globalement égale à la différence entre la masse minérale retenue dans l'accroissement courant de l'année en question et celle que retiendra ce même accroissement quand la composition de ses tissus sera stabilisée.

C'est-à-dire :

(4)

$$
\left(\begin{array}{c}
\text { minéralomasse } \\
\text { impliquée } \\
\text { dans les transferts } \\
\text { issus des bois } \\
\text { et écorce }
\end{array}\right]=\left(\begin{array}{c}
\text { masse minérale totale } \\
\text { nécessaire } \\
\text { à l'élaboration } \\
\text { des tissus ligneux } \\
\text { annuels } \\
\Leftrightarrow \begin{array}{c}
\text { prélèvement } \\
\text { apparent }
\end{array}
\end{array}\right)-\left[\begin{array}{c}
\text { masse minérale } \\
\text { immobilisée } \\
\text { annuellement } \\
\begin{array}{c}
\text { immobilisation } \\
\text { nette }
\end{array}
\end{array}\right]
$$

En terme de biomasse la quantité de tissus formée annuellement est équivalente (en matière sèche) à l'immobilisation et correspond à la somme des accroissements individuels de chaque compartiment.

En terme de minéralomasse ces deux grandeurs sont très différentes puisque le tissu âgé a une concentration stabilisée ( $\alpha$ st.) très inférieure à celle qu'il avait l'année de sa formation $(\alpha \mathrm{j}$.).

Entre $\alpha$ st. et $\alpha \mathrm{j}$. on peut écrire que :

$$
\alpha \text { st. }=\alpha \mathrm{j} .-\alpha \text { (transferts }+ \text { lessivage). }
$$

\section{Remarque :}

- L'évaluation que dous donnons pour $\alpha$ st. n'est pas toujours absolument rigoureuse dans la mesure où pour certains compartiments (branches par exemple) il n'est pas absolument certain que les transferts soient terminés : on fait dans ce cas une évaluation par défaut.

- La quantité d'éléments minéraux lessivés par les eaux atmosphériques à partir $\mathrm{du}$ bois et écorce est faible et impossible à séparer du pluviolessivage des feuilles; nous l'avons pris en compte dans le calcul des transferts issu des feuilles : on peut écrire $\alpha$ st. $=\alpha \mathrm{j}$. $-\alpha$ tr.

La minéralomasse retenue dans l'immobilisation nette annuelle s'écrit pour chaque compartiment $\operatorname{Im} \times \alpha$ st. 
La minéralomasse retenue dans les tissus formés la même année s'écrit pour chaque compartiment $\mathrm{Q} \times \alpha \mathrm{j}$.

D’après les remarques précédentes, l'expression (4) s'écrit :

$$
\operatorname{Im} \alpha \text { st. }=\mathbf{Q} \alpha \mathrm{j} .-\mathbf{Q} \alpha \mathrm{tr} .
$$

Les transferts issus des bois et écorce sont évalués d'après l'expression :

(5) $\operatorname{tr}(\mathrm{e}+\mathrm{b})=\mathrm{Q} \alpha \mathrm{j} .-\operatorname{Im} \alpha$ st.

Cette mise au point sur les phénomènes de transfert nous permet de proposer une expression plus générale que l'expression (2) dans le prélèvement net de bioéléments par un peuplement forestier :

Prélevement réel $=$ [masse d'éléments nécessaire à l'élaboration annuelle des tissus ligneux + masse d'éléments nécessaire à l'élaboration de la masse foliaire] — [transferts internes issus des parties ligneuses + transferts issus de la masse foliaire].

En pratique les transferts issus des feuilles sont évalués par l'expression (3), ceux issus des bois et écorce par l'expression (5).

Ces formules montrent limportance des transferts internes dans le cycle biologique des éléments nutritifs. Ils minimisent le prélèvement apparent des peuplements forestiers pour l'élaboration de la biomasse annuelle.

Si l'on considère «l'écosystème clos» la quantité d'éléments absorbés par le peuplement se limite à l'immobilisation.

Dans l'étude complète d'un bilan de l'écosystème il faut tenir compte de facteurs supplémentaires tels que le drainage d'éléments minéraux vers les couches profondes du sol, mais aussi de l'altération qui libère à partir des minéraux primaires des quantités d'éléments nutritifs capables vraisemblablement, sinon d'équilibrer, au moins de compenser partiellement les pertes et les exportations en conditions d'exploitation forestière classique.

\section{2. - Etude du cycle biologique simplifié dans les deux écosystèmes}

Nous étudierons les points suivants :

- l'immobilisation courante annuelle des peuplements,

- la restitution au sol par la chute de litière et le pluviolessivage,

- le calcul du prélèvement annuel, le problème des transferts,

- l'évaluation des réserves du sol et le drainage profond.

\subsection{1. - L'immobilisation courante annuelle des peuplements}

La détermination de cette donnée nécessite la mesure de la productivité nette des écosystèmes étudiés.

Rappel de définition :

Prélèvement réel : éléments prélevés effectivement du sol pour élaborer les tissus de l'accroissement annuel.

Prélèvement apparent : masse totale d'éléments retenue dans l'accroissement annuel de la biomasse, mais dont une partie seulement est prélevée au sol (le reste provient des transferts internes).

Quand on parle de prélèvement on entend toujours prélèvement réel. 
ב

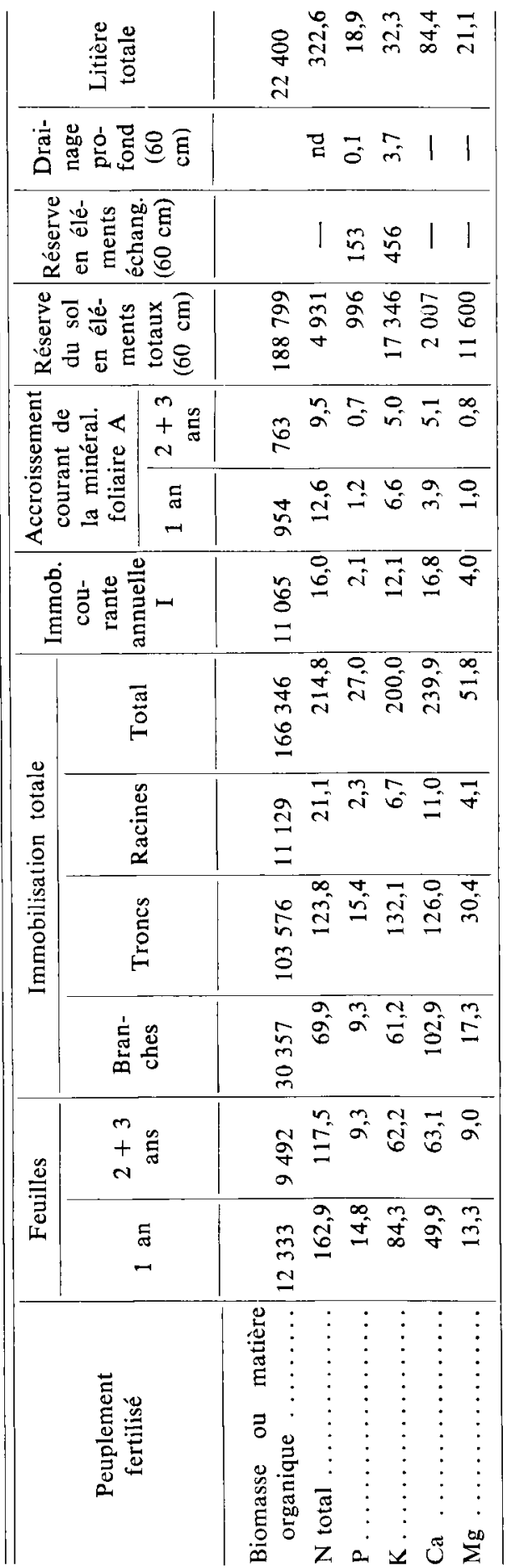

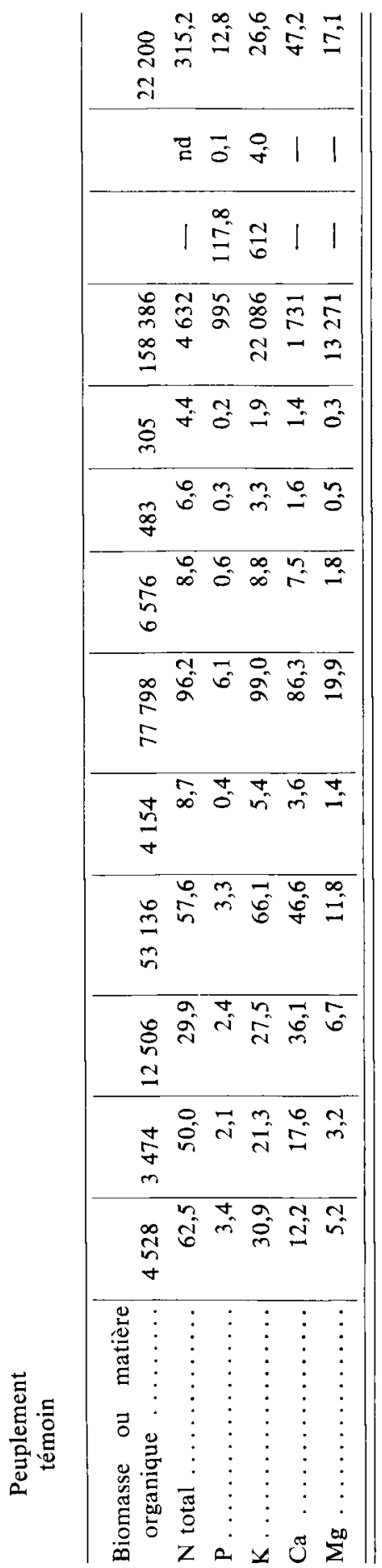



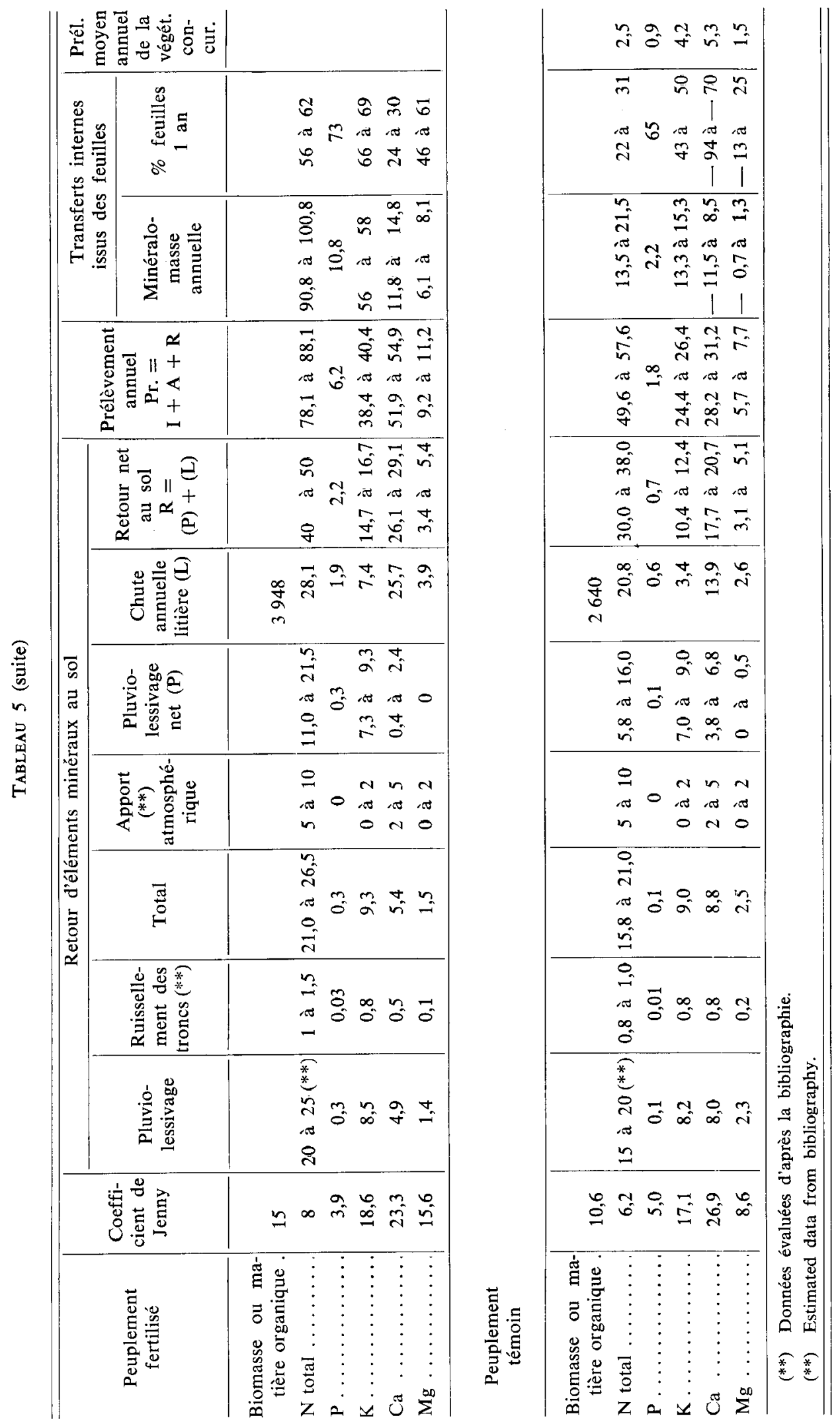
Les biomasses ont été mesurées en 1975, et des tarifs ont été établis. Nous ne les avons pas remesurées en 1978 mais nous les avons évalués en considérant en première approximation que dans un intervalle de temps assez court ( 3 ans) les tarifs calculés en 1975 restent valables. Pour un peuplement jeune, il est plus logique de raisonner en productivité courante qu'en productivité moyenne.

A partir d'un inventaire des circonférences en 1978, nous avons calculé la biomasse compartimentée de tous les arbres du placeau, les chiffres sont ramenés à l'hectare.

Remarque : pour avoir des chiffres comparables avec 1975 dans le placeau fertilisé on est obligé de calculer la biomasse 1978 en incluant l'éclaircie sanitaire de 1973, en lui affectant un accroissement moyen en surface terrière équivalent à celui du reste du peuplement, ce qui représente une approximation car l'accroissement s'est en partie, au moins, reporté sur les arbres restants.

Le tableau 6 montre l'évolution des biomasses forestières entre 15 et 18 ans. Pour les deux peuplements l'accroissement en matière sèche est important : 30 p. 100 pour le peuplement fertilisé, 40 p. 100 pour le témoin; l'accroissement des troncs ne montre pas un tel écart : 34 p. 100 dans le peuplement fertilisé contre 36 p. 100 dans le témoin. Il est possible cependant que le peuplement fertilisé perde un peu de son avantage initial.

TABLEAU 6

Biomasse totale et productivité des deux peuplements exprimées en $\mathrm{kg}$ de matière sèche par ha

Total biomass and productivity of the two stands (in $\mathrm{kg}$ of dry matter per ha)

\begin{tabular}{|c|c|c|c|c|c|c|}
\hline \multirow[b]{2}{*}{ Année } & \multicolumn{2}{|c|}{$\begin{array}{l}\text { Peuplement } \\
\text { fertilisé }(F)\end{array}$} & \multicolumn{2}{|c|}{$\begin{array}{l}\text { Peuplement } \\
\text { témoin }(\mathrm{T})\end{array}$} & \multicolumn{2}{|c|}{$\begin{array}{l}\text { Différence } \\
(\mathrm{F}-\mathrm{T})\end{array}$} \\
\hline & $\begin{array}{c}1978 \\
\text { (18 ans) }\end{array}$ & $\begin{array}{c}1975 \\
(15 \text { ans }))\end{array}$ & 1978 & 1975 & 1978 & 1975 \\
\hline Biomasse $\ldots \ldots \ldots \ldots \ldots$ & 166386 & 128040 & 77798 & 55707 & 88588 & 69475 \\
\hline $\begin{array}{c}\text { Accroissement moyen an- } \\
\text { nuel } \ldots \ldots \ldots \ldots \ldots \ldots\end{array}$ & 9244 & 8346 & 4322 & 3714 & 4922 & 4632 \\
\hline $\begin{array}{c}\text { Accroissement courant an- } \\
\text { nuel } \ldots \ldots \ldots \ldots \ldots \ldots \ldots\end{array}$ & 12782 & nd & 7364 & nd & 5418 & nd \\
\hline
\end{tabular}

La minéralomasse des peuplements en 1978 (tableau 7) a été évaluée en appliquant aux biomasses compartimentées de cette même année les concentrations moyennes mesurées en 1975. Le risque de biais le plus important est lié aux minéralomasses des organes annuels dans la mesure où les concentrations de l'année 1975 s'écarteraient trop de la moyenne.

L'immobilisation courante annuelle nette est la minéralomasse fixée définitivement dans l'accroissement courant annuel des parties ligneuses des biomasses : branches vivantes et mortes, troncs, racines. 


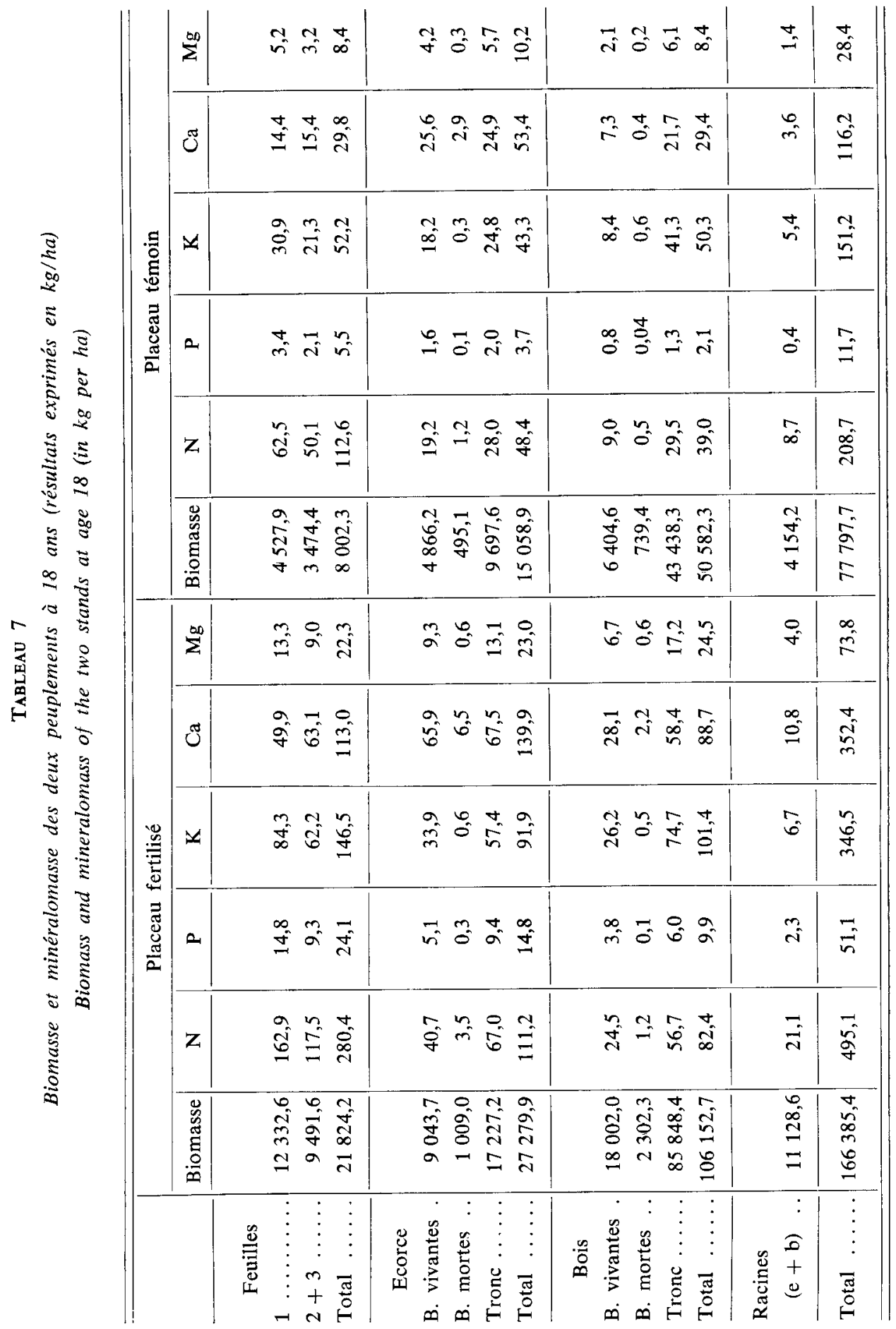




\subsection{2. - La restitution d'éléments minéraux au sol}

\subsection{1. - La restitution par les chutes de litière}

Les retombées annuelles de litière ne subissent pas de cycle régulier au cours des deux années étudiées (octobre 1976 à septembre 1978).

$\mathrm{Si}$ on relie les chutes de litière à la pluviométrie, on constate une certaine relation de l'automne 1977 à l'été 1978, par contre la première année de récolte ne montre pas de rapports étroits entre ces facteurs. Les chutes de litière pendant cette période sont plus liées aux aléas climatiques : tempête en avril 1977 et vents forts en septembre 1977. Il est possible en outre que le déséquilibre climatique de 1976 ait un rapport avec les fortes chutes de litière à la fin de cette année.

Au total, si un certain cycle annuel de chute de litière existe sur une période d'observation beaucoup plus longue, celui-ci peut être largement modifié par les facteurs climatiques à effet mécanique (tempête, orage...), ou à effet physiologique (sécheresse, humidité, température) comme l'on souligné Sarvas (1972) et MaLKÖNEN (1974). Le parallélisme de la dynamique du retour de litière dans les deux peuplements est remarquable. La différence d'intensité est liée à la différence de biomasse foliaire des deux peuplements.

La quantité moyenne annuelle de matière sèche arrivant au sol est de $3947 \mathrm{~kg} / \mathrm{ha}$ dans le peuplement fertilisé contre $2640 \mathrm{~kg} / \mathrm{ha}$, soit une différence de $1307 \mathrm{~kg} / \mathrm{ha}$ (tableau 8). Les écarts annuels sont importants et de même ordre de grandeur dans les deux peuplements ( $\pm: 25$ p. 100 de la moyenne).

\subsection{1. - Variation saisonnière de la composition minérale des litières}

D'une manière globale les teneurs en $\mathbf{P}, \mathbf{K}$ et $\mathbf{C a}$ sont plus élevées dans la litière du peuplement fertilisé que dans celle du témoin, e'est l'inverse pour l'azote et le magnésium : il y a donc une relation évidente entre la composition des litières et celle du matériel vivant. L'étude des variations saisonnières permet de tirer les conclusions suivantes :

- il n'y a pas de corrélation entre les quantités de litière et leur composition ;

- on constate un parallélisme assez remarquable entre les variations saisonnières des compositions chimiques des litières des deux peuplements;

- l'allure des variations relatives des compositions chimiques annuelles pour un élément donné est assez semblable; en absolu les teneurs varient d'une année à l'autre. L'allure des variations relatives des compositions chimiques saisonnières peut se résumer de la façon suivante :

- l'azote et le phosphore ont un comportement assez semblable avec des teneurs maximum au printemps et en été. Les variations du phosphore restent toutefois assez modestes par rapport à celles de l'azote ;

- le potassium et le magnésium ont également un comportement très semblable avec des teneurs maximum en été et secondairement en automne ;

- le calcium varie comme le magnésium dans le peuplement fertilisé et comme lazote dans le témoin. 


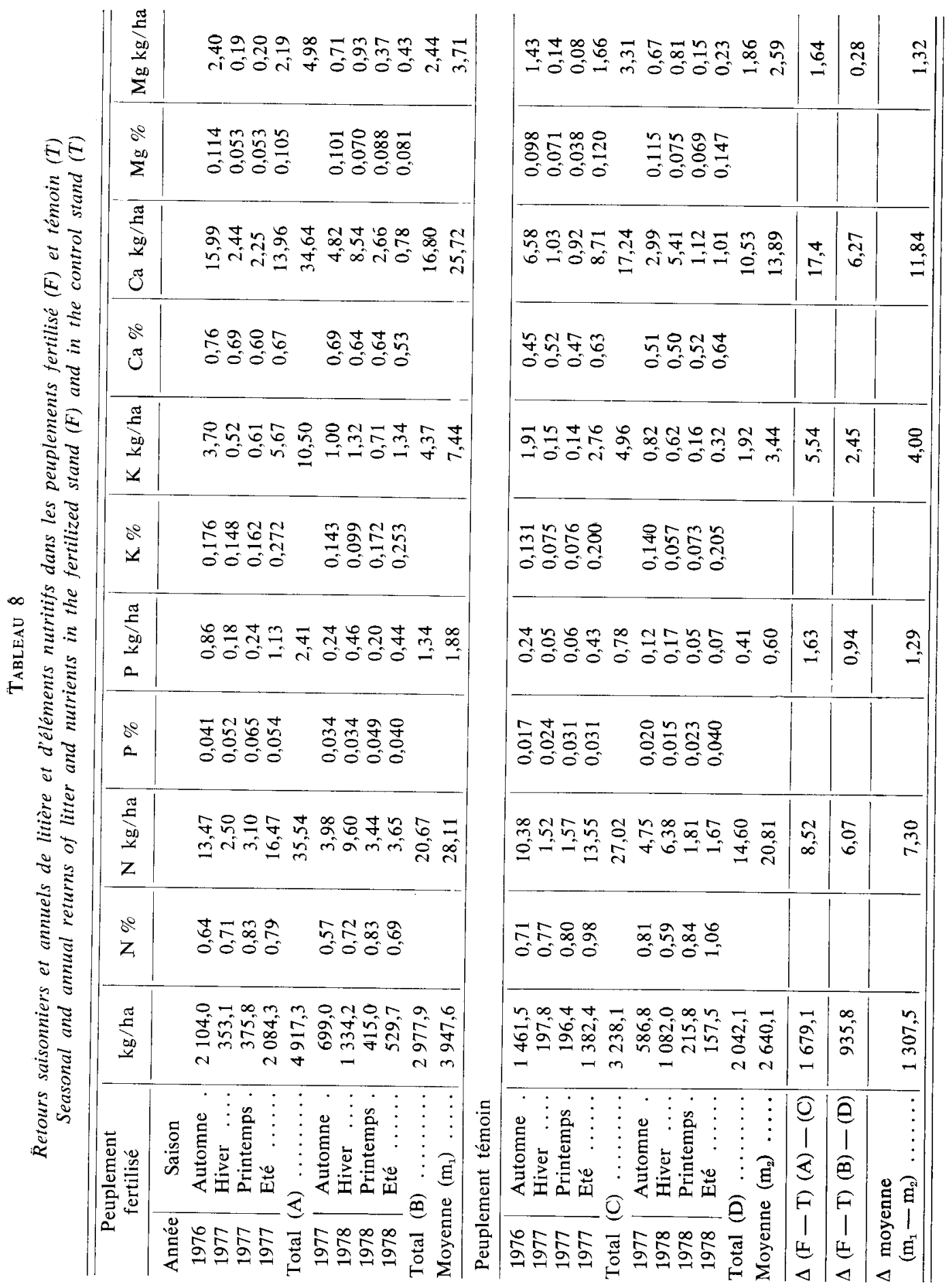




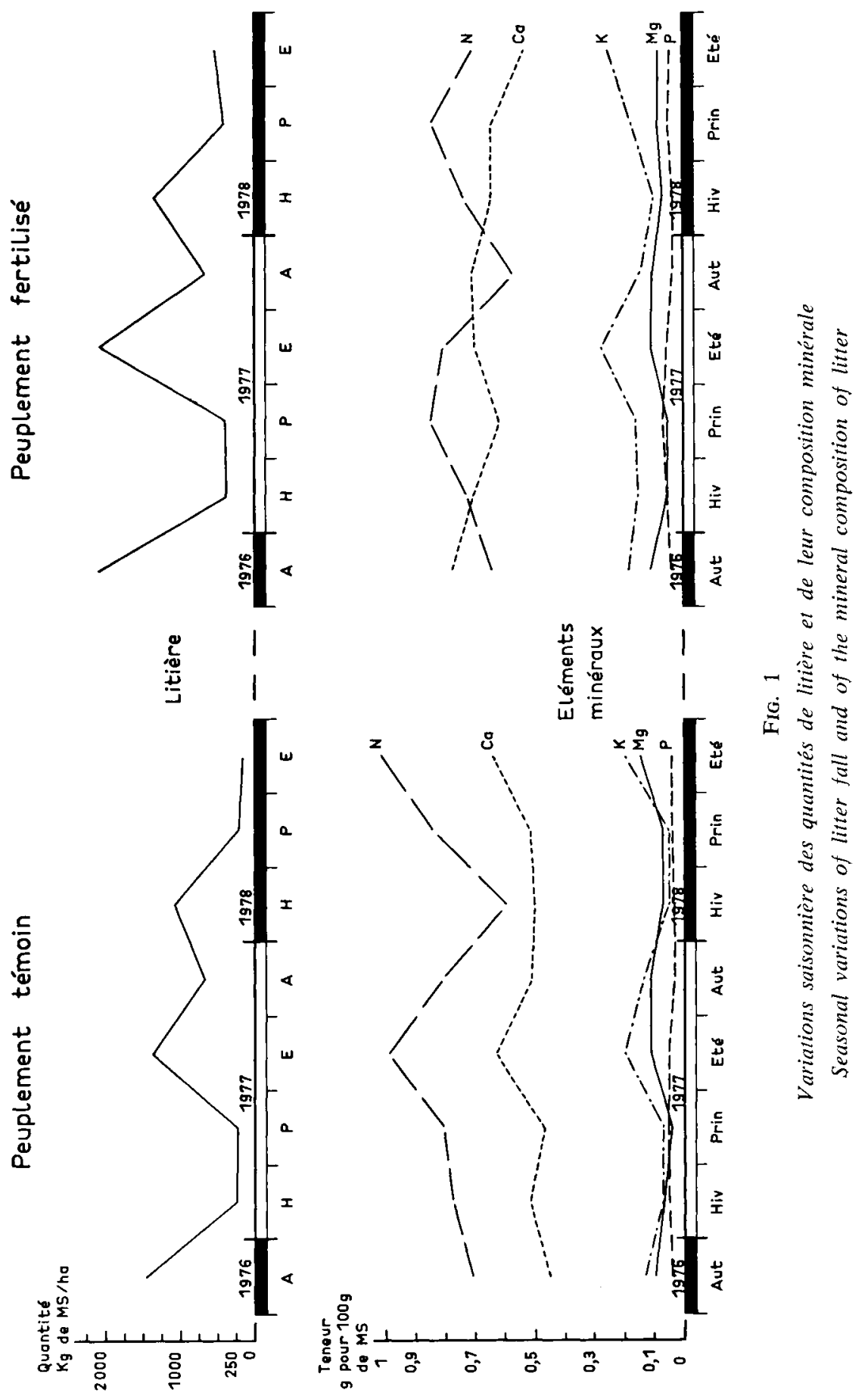




\subsection{2. - Variations saisonnières des quantités d'éléments nutritifs retournant au sol par la litière (tableau 8)}

L'observation des résultats montre d'une façon très nette la corrélation qui existe entre la quantité d'éléments nutritifs retournant au sol par l'intermédiaire des litières et la quantité de litière des peuplements. Ceci a déjà été signalé par AussENAC et al. (1972).

On va donc retrouver les mêmes conclusions que pour la litière :

- pas de cycle régulier annuel;

- parallélisme du comportement des deux peuplements.

On constate de plus une liaison étroite entre les retombées, les biomasses des peuplements et leur statut nutritif. L'intensité relative de la restitution des éléments minéraux est la suivante :

$$
\mathrm{N}>\mathrm{Ca} \gg \mathrm{K}>\mathrm{P}>\mathrm{Mg}
$$

Quantitativement les données pour les deux peuplements sont de l'ordre de celles obtenues par Aussenac et al. (1972) pour des peuplements résineux divers mais plus âgés dans l'est de la France.

Une comparaison plus intéressante peut être faite avec les données de MiLler et al. (1976) sur la même essence, mais en station différente (sables soufflés très pauvres, Ecosse) et dans un peuplement plus vieux (50 ans). La fertilisation à l'état adulte (34 ans) est destinée à tester l'effet de l'azote apporté à différentes doses (kg/ha) :

$$
(\mathrm{F} 1=0, \mathrm{~F} 2=84, \mathrm{~F} 3=168, \mathrm{~F} 4=336, \mathrm{~F} 5=504)
$$

avec une fertilisation de base dans tous les traitements $(21 \mathrm{~kg}$ de $P$ et $120 \mathrm{~kg}$ de $K /$ ha).

Les résultats sont consignés dans le tableau 9.

\section{TABLEAU 9}

Comparaison des retombées de litières et d'éléments nutritifs dans différentes stations (résultats exprimés en $\mathrm{kg} / \mathrm{ha} / \mathrm{an}$ )

Comparison of the litter and mutrients returns in various sites

\begin{tabular}{|c|c|c|c|c|c|c|c|}
\hline & & $\mathrm{N}$ & $P$ & K & $\mathrm{Ca}$ & $\mathrm{Mg}$ & Matière sèche \\
\hline Ecosse & $F_{1}$ & 11,0 & 1,8 & 3,3 & 13,6 & 1,9 & 2615 \\
\hline \multirow{3}{*}{$\begin{array}{r}\text { Pin laricio } \\
(50 \text { ans })\end{array}$} & & & & & & & \\
\hline & $F_{2}$ & 17,8 & 2,2 & 5.5 & 14,2 & 2,5 & 3136 \\
\hline & $F_{:}$ & 25,0 & 2,5 & 7,3 & 16,5 & 2,8 & 3657 \\
\hline \multirow[t]{2}{*}{ Miller (1976) } & $\mathrm{F}_{4}$ & 31,6 & 2,4 & 7,7 & 16,2 & 2,9 & 3837 \\
\hline & $F_{5}$ & 39,0 & 2,6 & 7,3 & 14,2 & 2,9 & 3970 \\
\hline Moulière & $\mathrm{F}$ & 28,1 & 1,9 & 7,4 & 25,7 & 3,9 & 3948 \\
\hline $\begin{array}{r}\text { Pin laricio } \\
(18 \text { ans })\end{array}$ & $\mathrm{T}$ & 20,8 & 0,6 & 3,4 & 13,9 & 2,6 & 2640 \\
\hline
\end{tabular}
(in kg per ha per year) 
Les traitements $F$ et $F 4$ ainsi que $T$ et $F 1$ sont comparables quant aux chutes de litière et aux quantités d'éléments nutritifs restitués. Il doit donc y avoir une compensation de facteurs car l'âge du peuplement leur confère des structures différentes.

\subsection{2. - Les restitutions par le pluviolessivage et le ruissellement des troncs}

Une partie des bioéléments est mobilisée directement par les eaux de pluie par entraînement soit à l'état dissous, soit à l'état de fines particules (on n'observe en fait que la résultante de phénomènes complexes car l'absorption directe d'éléments à partir des eaux de pluie existe également) (Tukey et al., 1952, in MaLkönen, 1974).

Il faut tenir compte du pluviolessivage net, c'est-à-dire du pluviolessivage mesuré sous le couvert végétal duquel on déduit les apports atmosphériques des eaux de pluie.

Les chiffres que nous avons obtenus ne sont qüindicatifs étant donné le faible nombre de pluviomètres disposés sous le couvert et qui n'ont été relevés que pendant une seule année (1977).

La pollution de notre pluviomètre témoin nous empêche de donner les résultats pour la composition des eaux de pluie ; nous ne donnerons qu'une évaluatior bibliographique d'après les données de Duvigneaud (1974), Rapp (1972), Miller (1963), Ulrich (1973), CARLISTE et al. (1967). Les apports correspondent à ceux dune zone rurale située à proximité d'un centre urbain. La restitution annuelle par le ruisscllement des trones n'a pas été mesurée. Nous avons admis que la restitution par ruissellement de troncs se faisait dans les mêmes proportions que celles mesurées par AussENAC et al. (1972) sur un peuplement résineux d’âge et de structure comparables.

Le pluviolessivage net du potassium est assez élevé; ceux du phosphore et du magnésium sont très faibles, le calcium a un comportement intermédiarre; ces données sont tout à fait similaires dans les deux peuplements.

\subsection{Calcul du prélèvement annuel des bioúléments majeurs} pour les deux peuplements

Le prélèvement annuel des éléments nutritifs majeurs a été calculé par la formule :

Prélèvement $=$ immobilisation + variation de la masse d'éléments nécessaires à l'accroissement des feuilles et radicelles + retour net.

Les résultats concernant le cycle biologique dans les deux peuplements sont consignés dans le tableau 5.

Le prélèvement des différents éléments est pratiquement deux fois plus fort dans le traitement fertilisé que dans le témoin, ce qui est très logique compte tenu des différences de biomasse des deux peuplements.

Pour le peuplement non fertilisé dont le couvert végétal n’est pas fermé, la minéralomasse de la végétation concurrente des arbres forestiers prélève annuellement 
une masse d'éléments non négligeable qui participe notablement au prélèvement annuel réel de la masse végétale totale.

Nous n'avons pas étudié le prélèvement courant annuel de la végétation concurrente, cependant on peut chiffrer le prélèvement moyen annuel.

Il faut être prudent dans l'interprétation des résultats car certains éléments peuvent avoir plusieurs cycles dans la même année (c'est le cas du potassium) et le prélèvement peut avoir ainsi un certain caractère apparent.

Nous pouvons comparer nos résultats à ceux obtenus par ULRICH (1973) et Wells et al. (1975) (tableau 10).

Tableau 10

Comparison of biological cycle in various ecosystems (data in $\mathrm{kg}$ per ha per year) (données exprimées en $\mathrm{kg} / \mathrm{ha} / \mathrm{an}$ )

Comparison of biological cycle in various ecosystems (datas in $\mathrm{kg}$ per ha per year)

\begin{tabular}{|c|c|c|c|c|c|c|c|}
\hline & & $\mathbf{N}$ & $\mathbf{P}$ & K & $\mathrm{Ca}$ & & $\mathbf{M g}$ \\
\hline \multirow{3}{*}{$\begin{array}{l}\text { Pessière de classe I } \\
\text { (ULRICH, 1973) }\end{array}$} & Immob. ... & 20 & 2,0 & 11 & 13 & & 2,2 \\
\hline & Retour ... & 48 & 4,4 & 28 & 24 & & 2,0 \\
\hline & Prélèv. .. & 78 & 6,4 & 39 & 37 & & 4,2 \\
\hline \multirow{3}{*}{$\begin{array}{l}\text { Pessière de classe IV } \\
\text { (ULRICH, 1973) }\end{array}$} & Immob. & 10 & 1,1 & 5 & 7 & & 1,2 \\
\hline & Retour & 24 & 2,2 & 14 & 12 & & 0,6 \\
\hline & Prélèv. & 34 & 3,3 & 19 & 19 & & 1,8 \\
\hline \multirow{3}{*}{$\begin{array}{c}\text { Pinus taeda (U.S.A.) } \\
16 \text { ans } \\
\text { (Wells et al., 1975) }\end{array}$} & Immob. & 14 & 2,0 & 11 & 8 & & 3,0 \\
\hline & Retour & 68 & 8,3 & 28 & 35 & & 8,9 \\
\hline & Prélèv. & 100 & 21,0 & 64 & 58 & & 17,0 \\
\hline \multirow{3}{*}{$\begin{array}{l}\text { Pin Laricio de Corse } \\
\text { fertilisé, } 18 \text { ans }\end{array}$} & Immob. & 16,0 & 2,1 & 12 & 17 & & 4,0 \\
\hline & Retour & 40 à 50 & 2,2 & 15 à 17 & 26 à 29 & 3,4 & t̀ $\quad 5,4$ \\
\hline & Prélèv. & 78 à 88 & 6,1 & 38 à 40 & 52 à 55 & 9 & à 11 \\
\hline \multirow{3}{*}{$\begin{array}{l}\text { Pin Laricio de Corse } \\
\text { non fertilisé, } 18 \text { ans }\end{array}$} & Immob. & 8,6 & 0,6 & 8,8 & 7,5 & & 1,8 \\
\hline & Retour & 30 à 38 & 0,7 & 10 à 12 & 18 à 21 & 3 & à 5 \\
\hline & Prélèv. & 50 à 57 & 1,8 & 24 à 26 & 28 à 31 & 6 & à 8 \\
\hline
\end{tabular}

Le peuplement fertilisé est comparable à la pessière de classe I de Ulrich, tandis que le témoin est comparable à la pessière de classe IV et au peuplement de Pinus taeda de Wells et al.

L'immobilisation est une donnée très importante à connaître et surtout le pourcentage que celle-ci représente des réserves assimilables en particulier pour les ions peu mobiles (P). Le calcium n'apparaît pas dans les réserves assimilables simplement parce que les résultats indiquent une quantité assimilable supérieure à la quantité totale; ce n'est pas surprenant en sols pauvres en calcium (on se référera aux quantités totales). 


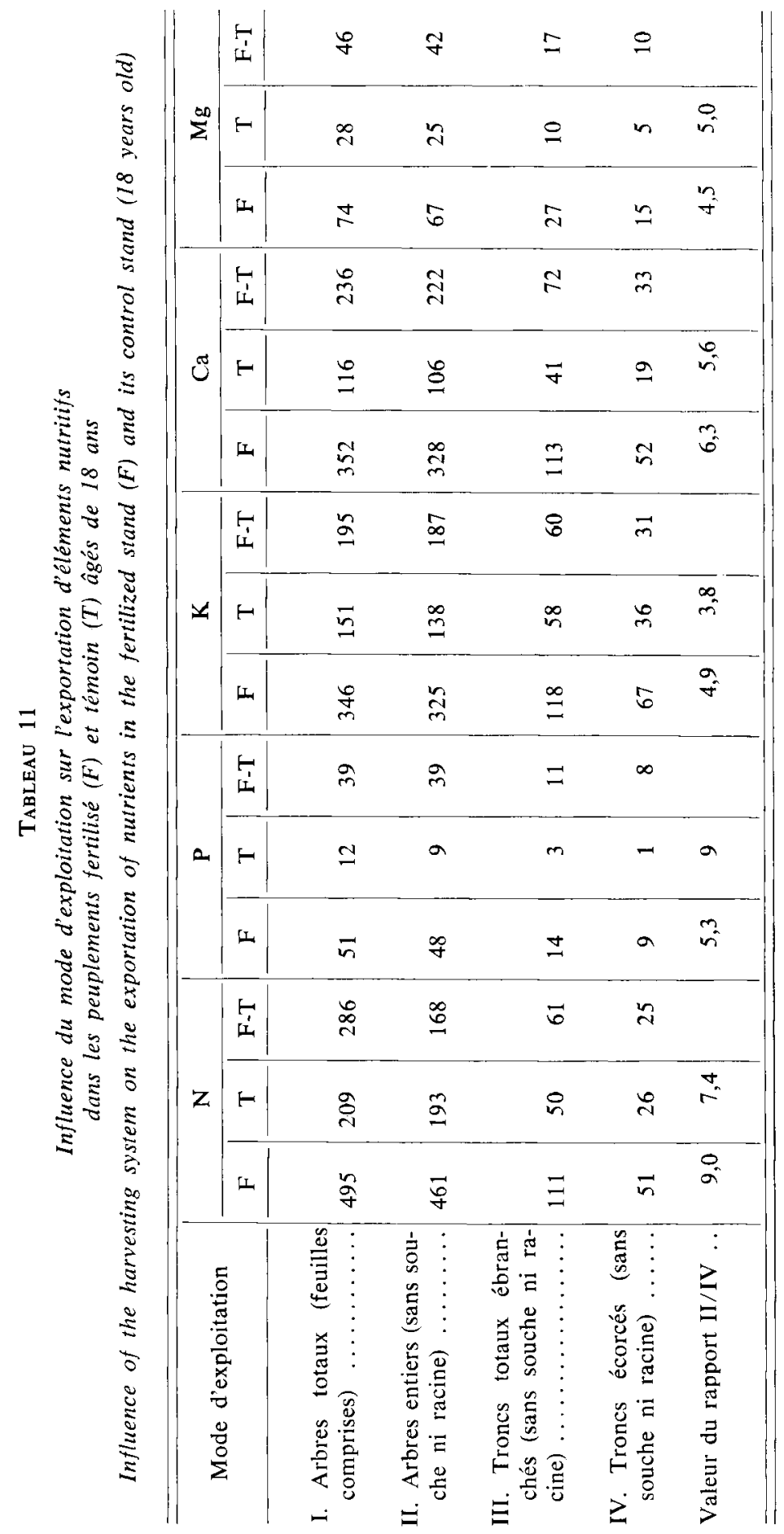


L'immobilisation dans les parties ligneuses (troncs, branches, racines) après 18 ans de végétation, calculée en pourcentage de réserves assimilables donne les résultats suivants :

Peuplement F : P 18, K 44, Ca 12.

Peuplement T: P 5, K 16, Ca 5 .

Cette immobilisation peut se transformer en exportation en fonction du mode d'exploitation (tableau 11). Nous avons calculé l'exportation hors de l'écosystème qu'entraîneraient les principaux types d'exploitation forestière envisageables.

Le rapport entre l'exportation par l'exploitation de l'arbre entier (sans racines) et celle de l'arbre écorcé est compris entre 4 et 9 suivant les éléments. L'exploitation de l'arbre entier produit une exportation considérable d'éléments nutritifs majeurs surtout si on la rapporte au stock d'éléments disponibles pour l'alimentation des plantes.

Pour un peuplement aussi jeune (18 ans), l'immobilisation dans les troncs ne représente qu'un faible pourcentage de la minéralomasse totale par rapport à un peuplement arrivant à maturité. La proportion relative des divers compartiments change considérablement avec l'âge du peuplement. SwITZER et al. (1968) ont montré que pour Pinus taeda la biomasse foliaire ne se stabilise que vers l'âge de 30 ans et celle des branches vers 40 à 50 ans.

\section{Le problème des transferts internes des bioéléments}

Wells \& Mrtz en 1963 évaluent ces transferts pour Pinus taeda et obtiennent les résultats suivants : 50 p. 100 pour $\mathrm{N}$ et $\mathrm{P}, 60$ p. 100 pour $\mathrm{K}$.

Wells \& Jörgensen (1975) sur la même essence les évaluent à 30 p. 100 pour $\mathrm{N}$, 0 p. 100 pour $P$ et 30 p. 100 pour K (les résultats sont exprimés en pourcentage de la masse d'éléments nécessaires à l'élaboration des feuilles de l'année).

Toujours pour Pinus taeda, Switzer et al. (1968) trouvent que les transferts internes fournissent 45 p. 100 des besoins annuels en azote. A partir des analyses des divers compartiments des arbres nous avons essayé d'apprécier ces transferts internes. Le problème est complexe car plusieurs phénomènes se superposent :

- la variation de composition effectivement liée aux transferts internes;

- la variation liée aux pluviolessivages;

- les différences dues aux variations annuelles de composition.

On constate que quel que soit le compartiment étudié (feuilles, bois, écorce) quand l'organe vieillit ( $1^{\text {re }}$ et $2^{*}$ année) la chute des teneurs en NPK est importante et comprise entre 10 et 50 p. 100 . Le phénomène inverse est observé pour le calcium (accumulation relative ou absolue). L'évolution des teneurs dépend de plus de la position relative du compartiment dans l'arbre. Pour les feuilles et les écorces, il est bien difficile de faire la part du pluviolessivage et des transferts, bien que l'azote et le phosphore soient peu entraînés par les eaux de pluie. Pour les bois, le lessivage ne doit intervenir que d'une façon minime, voire nulle.

Il est cependant difficile de retenir un chiffre pour évaluer les transferts car il faudrait tenir compte des transferts pour chaque compartiment en fonction du temps et de la représentation du compartiment dans la minéralomasse totale.

Les équations du paragraphe 5.1 nous permettent cependant de donner une évaluation de ces transferts (tableau 12). 
ปัป

\begin{tabular}{|c|c|c|c|c|c|c|c|c|c|c|}
\hline \multirow{3}{*}{ 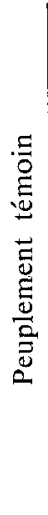 } & $\forall$ & $\begin{array}{l}\infty \\
\infty \\
\infty\end{array}$ & है & $\infty$ & $\begin{array}{l}m \\
2 \\
m \\
m \\
m\end{array}$ & 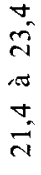 & $\stackrel{\infty}{\sigma}$ & 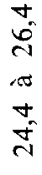 & $\begin{array}{l}0 \\
g \\
\dot{f} \\
\infty \\
\infty \\
\dot{J}\end{array}$ & $\begin{array}{l}a \\
\text { o } \\
\infty \\
= \\
=\end{array}$ \\
\hline & 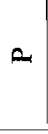 & $b_{0}^{0}$ & $\stackrel{6}{-1}$ & $\stackrel{\leftrightarrow}{G}$ & î & $\stackrel{r}{m}$ & in & $\stackrel{\infty}{\sim}$ & $\begin{array}{l}0 \\
\text { f̊ }\end{array}$ & $\begin{array}{l}\infty \\
\stackrel{2}{\Omega} \\
\underline{n}\end{array}$ \\
\hline & $\mathbf{z}$ & $\underbrace{\infty}_{\infty}$ & $\begin{array}{l}0 \\
\text { on }\end{array}$ & $\stackrel{\nabla}{\ominus}$ & 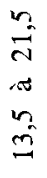 & 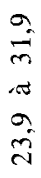 & $\frac{n}{\infty}$ & $\begin{array}{l}0 \\
i \\
n \\
6 \\
9 \\
q\end{array}$ & $\begin{array}{l}\vec{m} \\
\text { aे } \\
\text { m} \\
\text { gi }\end{array}$ & $\begin{array}{l}m \\
\vec{J} \\
\infty \\
\vec{v} \\
\vec{v}\end{array}$ \\
\hline \multirow{3}{*}{ 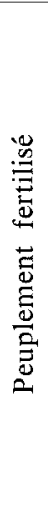 } & 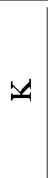 & $\overrightarrow{\mathrm{I}}$ & $\stackrel{n}{m}$ & $\Rightarrow$ & $\begin{array}{l}0 \\
\infty \\
n \\
\pi \\
0 \\
0 \\
n\end{array}$ & $\begin{array}{l}\vec{R} \\
R \\
R\end{array}$ & $\begin{array}{l}2 \\
\equiv \\
=\end{array}$ & 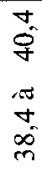 & $\begin{array}{l}n \\
6 \\
6 \\
0 \\
6\end{array}$ & 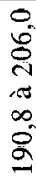 \\
\hline & $A$ & $\bar{i}$ & $\stackrel{\infty}{+}$ & $\bar{i}$ & $\hat{\theta}$ & $\stackrel{+}{g}$ & $\begin{array}{l}6 \\
\stackrel{0}{-}\end{array}$ & గె & $\begin{array}{l}\sigma_{0} \\
\infty \\
0\end{array}$ & $\begin{array}{l}\overrightarrow{0} \\
\overrightarrow{0}\end{array}$ \\
\hline & $\mathbf{Z}$ & $\underline{0}$ & $\stackrel{m}{m}$ & $\stackrel{m}{n}$ & $\begin{array}{l}\infty \\
8 \\
\delta \\
\infty \\
\infty \\
0 \\
0\end{array}$ & $\begin{array}{l}\overrightarrow{6} \\
= \\
0 \\
0\end{array}$ & 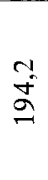 & $\begin{array}{l}\vec{\infty} \\
\infty \\
\stackrel{\infty}{0} \\
\stackrel{0}{1}\end{array}$ & 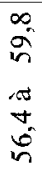 & 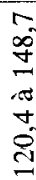 \\
\hline
\end{tabular}

(3)
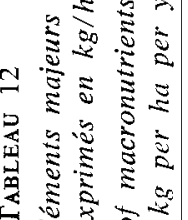

20

오

胥.

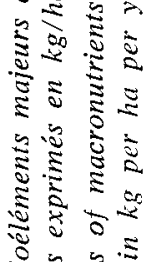

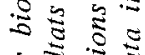

ช.

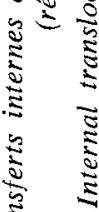

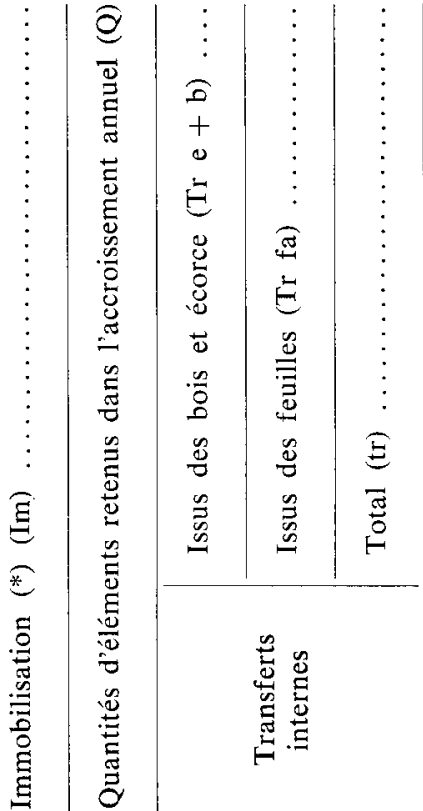

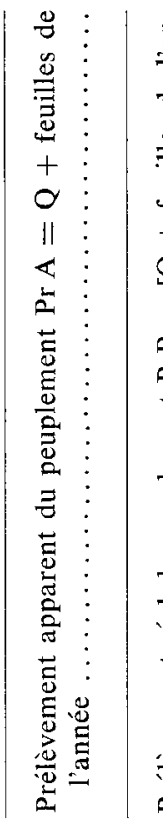

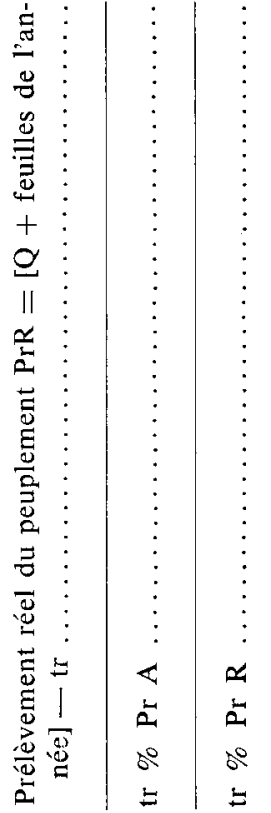

$\dot{-1}$ 
Les résultats obtenus pour les transferts issus des feuilles indiquent que leur amplitude est voisine de celle du prélèvement dans le peuplement fertilisé, alors qu'elle n'est que de la moitié de celui du témoin (sauf toutefois pour le phosphore). Pour ce dernier le schéma des transferts pour les différents éléments est classique; on trouve en effet un transfert important pour NPK, faible pour $\mathrm{Mg}$ et nul pour $\mathrm{Ca}$. Dans le peuplement fertilisé on retrouve le transfert important pour NPK, mais de plus un transfert non négligeable pour $\mathrm{Ca}$ et $\mathrm{Mg}$.

Ce transfert positif de calcium pose un problème. Si on se réfère à l'évolution des concentrations des feuilles avec le temps, on constate qu'elles s'enrichissent en calcium, cependant la teneur en $\mathrm{Ca}$ des litières est toujours plus faible que celle des feuilles les plus âgées récoltées sur l'arbre; il est possible qu'au moment de l'abscission une certaine quantité de calcium migre vers les tiges. On peut mettre ce phénomène en parallèle avec l'évolution des teneurs des écorces qui s'enrichissent en $\mathrm{Ca}$ en vieillissant puis s'appauvrissent au moment où la nécromasse l'emporte. De toute façon il n'y a peut-être pas incompatibilité entre transfert et accumulation à partir d'un même organe.

Nous avons vu dans les généralités que les transferts issus des bois et écorce n'entrent pas en compte dans la formulation du prélèvement des bioéléments et par là même dans l'expression des transferts internes qui en est issue, ces derniers représentant la quantité d'éléments minéraux migrant des feuilles âgées vers les jeunes pousses.

Nous avons établi une formule permettant d'évaluer les transferts issus des bois et écorce (formule 5, $\$ 5.1$.). Dans les deux peuplements l'ordre de grandeur de ces transferts est assez voisin de celui de limmobilisation. Les transferts totaux, issus des feuilles, bois et écorce, calculés par rapport au prélèvement annuel apparent (qui exprime la quantité totale de bioéléments contenue dans les tissus de l'année) représentent un fort pourcentage de celui-ci (entre 30 et 65 p. 100). On constate donc que ces phénomènes de transfert participent largement à l'économie d'éléments nutritifs du système par leur recyclage massif des parties sessiles vers les organes en croissance.

\subsection{4. - Evaluation des réserves du sol et du drainage annuel}

Les réserves du sol sont données pour les comparer au prélèvement annuel et à l'immobilisation des peuplements. Le drainage a été mesuré par une méthode connue (lysimétrie) mais qui est discutable. En effet, les échantillons sont placés dans un contexte assez différent du sol en place : drainage latéral, prélèvements et retours éliminés, régimes thermiques et hydriques modifiés.

Les résultats obtenus pour le phosphore et le potassium sont d'un ordre de grandeur raisonnable, ce qui n'est pas le cas du magnésium et surtout du calcium. Les conditions artificielles doivent avoir une influence particulière sur la migration de ces deux éléments.

\section{6. - Synthèse et conclusion}

L'évaluation des minéralomasses compartimentées des peuplements forestiers et l'étude du cycle des éléments biogènes constituent les outils indispensables de gestion des écosystèmes forestiers. 
L'étude détaillée de ces facteurs est très complexe, très longue et de plus sujette à incertitude.

Le matériel végétal pérenne est soumis d'une part à un rythme lié aux caractères moyens de l'écosystème (sol, climat), mais aussi à un rythme plus aléatoire lié à la variabilité annuelle de certains facteurs (atmosphérique et biotique).

Les mesures ne portent, en général, que sur quelques années au plus, si bien que si les facteurs annuels présentent un écart trop grand par rapport à la moyenne générale établie sur toute la durée de la révolution, ils introduisent dans les mesures des biais très difficiles à évaluer. Ce sont ces raisons qui font que les dynamiques globales sont très comparables entre les deux écosystèmes étudiés, mais que les cycles annuels sont assez variables. Les mesures obtenues sur quelques années représentent cependant une approche suffisante des phénomènes, au moins pour le but que nous nous étions fixé.

L'évaluation des minéralomasses nous a permis de mesurer l'immobilisation de deux peuplements depuis la plantation (18 ans) : pour le peuplement fertilisé (résultats par ha) on trouve $215 \mathrm{~kg}$ de $\mathrm{N}, 27 \mathrm{~kg}$ de $\mathrm{P}, 200 \mathrm{~kg}$ de K, $240 \mathrm{~kg}$ de Ca, $52 \mathrm{~kg}$ de $\mathrm{Mg}$; pour le peuplement témoin, $96 \mathrm{~kg}$ de $\mathrm{N}, 6 \mathrm{~kg}$ de $\mathrm{P}, 99 \mathrm{~kg}$ de $\mathrm{K}, 86 \mathrm{~kg}$ de Ca, $20 \mathrm{~kg}$ de $\mathrm{Mg}$. Exprimés par rapport aux ressources assimilables actuelles des sols, nous avons obtenu des chiffres élevés, en particulier pour le phosphore et le potassium (dans le peuplement fertilisé 18 p. 100 pour $P, 44$ p. 100 pour $K$; dans le témoin 5 p. 100 pour $\mathrm{P}, 16$ p. 100 pour K). On constate d’ailleurs que le peuplement fertilisé, malgré le doublement de la biomasse par rapport au témoin a un rendement très inférieur, en particulier pour l'utilisation du phosphore (il faut $0,23 \mathrm{~kg}$ de $P$ pour fabriquer $1 \mathrm{~m}^{3} \mathrm{de}$ bois de tronc dans le peuplement fertilisé, contre 0,11 dans le témoin). Il est difficile de savoir s'il s'agit réellement d'une consommation de luxe sans connaître l'optimum nutritif.

L'immobilisation peut se transformer en exportation en fonction du mode d'exploitation. En exploitation traditionnelle, les risques de perte de fertilité d'une station sont faibles d'autant que nos calculs ne tiennent pas compte de l'altération qui libère vraisemblablement des quantités d'éléments au moins égales à l'immobilisation dans les trones pendant la durée de la révolution. Par contre, l'exploitation de l'arbre entier exporte une quantité beaucoup plus importante d'éléments nutritifs. Si on ne restitue pas au sol les éléments non apportés par les pluies et pour lesquels les stocks assimilables sont faibles (phosphore et potassium), on risque d'être confronté rapidement à des problèmes de diminution sensible de production.

Les comparaisons que nous avons faites entre les modes d'exploitation portent sur des peuplements jeunes (18 ans) pour lesquels les résultats sont spectaculaires car les trones ne représentent qu'un faible pourcentage de la biomasse totale. La part relative du tronc ne va pas cesser d'augmenter jusqu'à la récolte, cependant même si la masse d'éléments des petits compartiments varie relativement peu jusqu'à cette échéance, elle n'en sera pas pour autant négligeable. Il faudra tenir compte en fin de révolution de la minéralomasse des litières qui représentent une immobilisation importante. Les éléments exportés devront être restitués sous forme d'engrais. Il sera très important de pouvoir chiffrer le gain de matière sèche que provoque l'exploitation totale de l'arbre et de le rapporter aux exportations supplémentaires qu'il occasionne. Les résultats obtenus pour un peuplement jeune ont donc une portée limitée mais ils peuvent devenir plus proches de la réalité dans le cas d'exploitation 
de peuplements à courte révolution. Il ne faut pas négliger non plus les exportations qu'entraînent les éclaircies. Dans les peuplements que nous avons étudiés la table de production de Decourt (1973), pour le Pin laricio de Sologne, prévoit un enlèvement de 20 p. 100 du volume à 20 ans.

Enfin, d'un point de vue plus théorique, il est intéressant d'évaluer le prélèvement annuel des peuplements et d'étudier l'influence de la fertilisation sur le cycle biologique. Le doublement de la production primaire (incluant l'éclaircie sanitaire du peuplement fertilisé) provoque un prélèvement pratiquement deux fois supérieur si l'on traite à part le cas du phosphore qui semble avoir un comportement particulier étant donné les très faibles teneurs qu'avait le sol avant la fertilisation.

L'évaluation des transferts internes, qui représente une économie naturelle très importante d'éléments nutritifs recyclés des parties âgées vers les organes en croissance, explique partiellement la frugalité des peuplements forestiers qui croissent sur des sols souvent très pauvres.

Reçu pour publication en octobre 1980.

\section{Remerciements}

Les relevés pluviométriques journaliers nous ont aimablement été fournis par M. RousSEAU, Chef de Secteur à l'Office national des Forêts.

\section{Summary \\ Study of mineralomass and biological cycle in two stands of corsican pine, fertilized or not at plantation}

This paper gives the results of a study of fertilization influence on biomass, mineralomass and nutrient cycle on a young coniferous stand in west of France.

Two ecosystems are compared : a corsican pine plantation (Pinus nigra Arn ssp laricio Poiret) fertilized or not at plantation. Besides static data like biomass, mineralomass, soil reserves, we try to give dynamic data : flows between the various compartments of the ecosystem, current annual immobilization of such stands, restitution to the soil by litter fall and throughfall real current annual amounts of bioelements required by trees (some special care has been taken in the evaluation of the internal bioelement translocation). 


\section{Références bibliographiques}

Arnett C., Eno L.F., Pritchett W.L., Fisher R.F., Garbett W.S., 1977. Annual report of cooperative research in forest fertilization.

Aussenac G., Bonneau M., Le Tacon F., 1972. Restitution des éléments minéraux au sol par l'intermédiaire de la litière et des précipitations dans quatre peuplements forestiers de l'Est de la France. CEcol. Plant., 7, 1-21.

Bonneau M., 1963. Premiers résultats d'une expérience de fertilisation sur Pin laricio de Corse en forêt de Moulière (86). Ann. Ec. Natl. Eaux Forêts Rech. Exp. for., XX, 3, 314-341.

Bonneau M., 1970. Résultats après 9 ans de l'essai de fertilisation de Moulière (86). Ann. Sci. forest., 27 (2), 111-125.

Bringmark L., 1977. A bioelement budget of an old scots pine forest in Central Sweden. In nutrient cycle in tree stands - Nordic Symposium. Silva fenn., 11 (3), 201-209.

Carlisle A., Brown A.H.F., White E.S., 1967. The nutrient content of rainfall and its role in the forest nutrient cycle. XIV Congrès I.U.F.R.O., Munich, II, 145-148.

Clement A., Janin G., 1973. Etude complémentaire de la présence de cristaux de carbonate de calcium dans le bois de peuplier; existence de cinq zones fonctionnelles reconnues à partir de leur teneur en phosphore. Ann. Sci. Forest., 30 (1), 63-81.

Decourt N., 1973. Tables de production pour les forêts françaises, édition E.N.G.R.E.F.

Duchaufour Ph., Bonneau M., 1959. Une méthode nouvelle de dosage du phosphore assimilable dans les sols forestiers. Bull. A.F.E.S., 4, 193-198.

Duvigneaud P., 1974. La synthèse écologique. Doin éd., 296 p.

Duvigneaud P., Denaeyer De Smets, 1964. Le cycle des éléments biogènes dans l'écosystème forêt. Lejeunia, 28, 1-148.

MALKöNEN E., 1974. Annual primary production and nutrient cycle in some scots pine stands. Metsantutkimuslaitoksen Julkaisusa.

Miller R.B., 1963. Plant nutrients in hard beech. II. The immobilisation of nutrients. III. The cycle of nutrients. Nz. J. Sci., 6, 365-413.

Miller H.G., CoOper J.M., Mili.er J.D., 1976. Effect of nitrogen supply on nutrients in litter fail and crown leaching in a stand of Corsican pine. J. Appl. Ecol., 13, 233-248.

Ovington J.D., 1965. Organic production turnover and mineral cycling in woodlands. Biol. Review, 40, 295-336.

Ovington J.D., 1971. Some factors affecting nutrient distribution within ecosystems. Colloque U.N.E.S.C.O., Bruxelles, 95-105.

Ranger J., 1977. Recherches sur les biomasses comparées de deux plantations de Pin laricio de Corse avec ou sans fertilisation. Ann. Sci. Forest., 35 (2), 93-115.

RAPP M., 1967. Production de litière et apport au sol d'éléments minéraux et d'azote dans un bois de Pin d'Alep (Pinus Halepensis Mill.). Ecol. Plant., 2, 325-338.

Rapp M., 1969. Production de litière et apport au sol d'éléments minéraux dans deux écosystèmes méditerranéens : la forêt de Quercus Ilex L. et la garrigue de Quercus coccifera L. Ecol. Plant., IV, 377-410.

RapP M., 1969. Apport d'éléments minéraux au sol par les eaux de pluviolessivage sous des peuplements de Quercus ilex L., Quercus lanuginosa et Pinus halepensis mill. Ecol. Plant., IV, 71-92.

RAPP M., 1971. Cycle de la matière organique et des éléments minéraux dans quelques écosystèmes méditerranéens. Dynamique de deux sols en climat tempéré, éd. C.N.R.S.

RENNIE P.J., 1955. The uptake of nutrients by native forest growth. Plant Soil, VII (1), 49-95. 
Rodin L.E., Bazilevich N.I., 1967. Production and mineral cycling in terrestrial vegetation. Oliver and Boyd, Edinburgh, 288 p.

SARVAS R., 1972. Investigations on the annual cycle of development of forest trees. Comm. Inst. for. fenn., 76 (3).

Switzer G.L., Nelson L.E., Smith W.H., 1968. The mineral cycle in forest stands. Forest and fertil. Theory and practice, Symposium Gainesville, 1967, published Tenessee Valley Authorithy.

Tukey H.B., Ticknor R.L., Hinsvark O.N., WitTwer S.H., 1952. Absorption of nutrients by stem branches of woody plants. Science, 116, 167-168.

UlRich B., 1973. Influence de la fertilisation sur le cycle des éléments nutritifs dans les écosystèmes forestiers. Symposium Int. F.A.O./I.U.F.R.O. sur l'utilisation des engrais en forêt. C.R. Minist. agric., 23-34.

Wells C.G., JoRgenSEN J.R., 1975. Nutrient cycling in loblolly pine plantations in Forest Soils and Forest land management. Les presses de l'Université Laval, Québec, 137-158.

Wells C.G., Metz L.S., 1963. Variation in nutrient content of Loblolly pine needles with season, age, soil and position on the crown. Soil. Sc. Amer. Proc., 27, 90-93.

Young H.E., Carpenter P.N., Altenberger, 1965. Preliminary tables of some chemical elements in seven trees species in Maine. Maine agric. Exp. St. techn. Bull., 20, University of Maine. 